\title{
Liderazgo integral educativo innovador en las escuelas normales superiores de Colombia ${ }^{1}$
}

\author{
Innovative Integral Educational Leadership in the Superior Normal Schools of Colombia ${ }^{2}$
}

\section{Liderança Educacional Integral Inovadora nas Escolas Normais Superiores da Colômbia ${ }^{3}$}

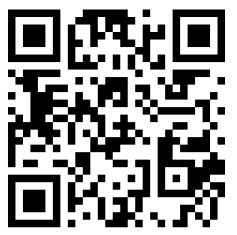

\author{
Amparo Novoa-Palacios \\ Universidad de La Salle \\ Bogotá, Colombia \\ amnovoa@unisalle.edu.co
}

https://orcid.org/0000-0002-6912-658X

Recibido $\cdot$ Received $\cdot$ Recebido: 09 / 11 / 2018
Corregido $\cdot$ Revised $\cdot$ Revisado: 12 / 03 / 2020
Aceptado $\cdot$ Accepted $\cdot$ Aprovado: 07 / 07 / 2020

Resumen: El artículo presenta la experiencia de 11 personas coordinadoras de las escuelas normales superiores de Colombia (ENS) que participaron en el Diplomado en Innovación Social Educativa. El objetivo que se persigue es indagar sobre la percepción que tienen acerca del liderazgo educativo desde los valores y la innovación. La metodología que se emplea es la sistematización de experiencias, con un enfoque cualitativo que articula la investigación descriptiva y el método de investigación y análisis documental. Los hallazgos resultan del análisis conceptual, comparativo y relacional de los aportes de las 11 personas líderes-coordinadoras de las Escuelas Normales Superiores, donde se identificaron algunos aspectos que caracterizan al sujeto líder integral educativo innovador como alguien que contribuye a la solución de los problemas socioeducativos, a partir de respuestas situadas en el territorio y una pertenencia que integra las diferentes esferas de la experiencia vital. Ello supone priorizar, desde un marco axiológico, el diálogo, la solidaridad y el asumir la tensión y el conflicto como parte del aprendizaje continuo. Igualmente se identifican que el territorio, los proyectos educativos y el fortalecimiento de competencias de carácter comunitario se constituyen en aspectos que hacen de un sujeto líder integral educativo innovador alguien capaz de conjugar las metodologías propias de la comunidad a partir del conocimiento profundo de los propios problemas.

Palabras claves: Liderazgo; valores; integral; educación; innovador.

\footnotetext{
1 Este artículo de investigación es resultado del proyecto de investigación Innovación en la formación inicial de maestros en contextos rurales en Colombia: Normales superiores de Gigante, Huila y María Montessori en Bogotá". Universidad de La Salle. CIV15101 (Novoa-Palacios, 2016).

${ }^{2}$ This research article is the result of the research project "Innovation in the initial training of teachers in rural contexts in Colombia: Normal Schools of Gigante, Huila, and María Montessori in Bogotá." Universidad de La Salle. CIV15101 (Novoa-Palacios, 2016).

${ }^{3}$ Este artigo de pesquisa é o resultado do projeto de pesquisa Inovação na formação inicial de professores em contextos rurais na Colômbia: superiores de Gigante, Huila e María Montessori em Bogotá." Universidad de La Salle. CIV15101 (Novoa-Palacios, 2016).
} 
http://doi.org/10.15359/ree.24-3.2

http://www.una.ac.cr/educare

educare@una.ac.cr

Abstract: The article analyzes the experience of eleven coordinators from the Superior Normal Schools of Colombia (ENS). They enrolled in the Educational Social Innovation Program. The goal is to study their perception of educational leadership based on values and innovation. The methodology used is the systematization of experiences, with a qualitative approach that articulates descriptive research and the method of research and documentary analysis. The results were obtained through the conceptual, comparative, and relational analysis of eleven leaders-coordinators' contributions from the Superior Normal Schools. In these institutions, the analysis identified some aspects characterizing the innovative educational integral leader as someone who contributes to solving socio-educational problems. These can be solved from responses located in the territory and from a sense of belonging that integrates the different spheres of life experience. This means prioritizing dialogue and solidarity and assuming tension and conflict as part of ongoing learning from an axiological framework. Likewise, the analysis found that the territory, the educational projects, and the strengthening of community-based competencies constitute aspects that make an integral, innovative, educational leader someone capable of combining community methodologies based on in-depth knowledge of the communities' problems.

Keywords: Leadership; values; integral; education; innovative.

Resumo: O artigo apresenta a experiência de onze pessoas coordenadoras das Escolas Normais Superiores da Colômbia (ENS) que participaram no Técnico em Inovação Social Educacional. O objetivo buscado é investigar a percepção de liderança educacional baseada em valores e inovação. A metodologia utilizada é a sistematização de experiências, com uma abordagem qualitativa que articula a pesquisa descritiva, o método de pesquisa e análise documental. Os resultados foram da análise conceitual, comparativa e relacional das contribuições de 11 líderes coordenadores das Escolas Superiores Normais, onde foram identificados alguns aspectos que caracterizam a pessoa líder integral educacional inovador como alguém que contribui para a solução de problemas socioeducativos, a partir de respostas localizadas no território e um sentido de pertencentes que integram as diferentes esferas da experiência de vida. Isso significa priorizar o diálogo, a solidariedade e assumir tensão e conflito como parte do aprendizado contínuo a partir de uma estrutura axiológica. Da mesma forma, identifica-se que o território, os projetos educacionais e o fortalecimento das competências comunitárias constituem aspectos que tornam um líder educacional integral inovador alguém capaz de combinar as metodologias próprias da comunidade com base no conhecimento profundo de seus próprios problemas.

Palavras-chave: Liderança; valores; integral; educação; inovador.

\section{Introducción}

El presente artículo tiene como objetivo indagar sobre la percepción que tienen las personas coordinadoras de 11 escuelas normales superiores de Colombia acerca del liderazgo en contexto educativo y que han participado en el Diplomado en Innovación Social Educativa

\footnotetext{
2 Amparo Novoa-Palacios

Los artículos de la Revista Electrónica Educare del Centro de Investigación y Docencia en Educación de la Universidad Nacional, Costa Rica, se comparten bajo términos de la Licencia Creative Commons: Reconocimiento, № Comercial, Sin 0bra Derivada 3.0 Costa Rica. Las autorizaciones adicionales a las aquí delimitadas se pueden obtener en el correo: educare@una.cr
} 
(ISE) ${ }^{4}$ durante un año en la Universidad de la Salle de Bogotá. Este Diplomado forma parte del proyecto de investigación sobre la innovación en la formación inicial de maestros en contextos rurales (Novoa-Palacios, 2016).

Estas Escuelas, según la Ley 115 de 1994, se definen de la siguiente manera:

Instituciones educativas con una unidad de apoyo académico. Es decir, son colegios que ofrecen desde el grado preescolar hasta [grado] $11 \mathrm{y}$, adicionalmente, cuentan con un Programa de Formación Complementaria (PFC) para la preparación de docentes que atienden preescolar y básica primaria. [Son] instituciones [que] han cumplido un papel preponderante en la educación colombiana en los territorios más apartados, con población dispersa y rural. (Mineducación, 2018, párr. 2-3)

Para abordar su desarrollo se plantea, en un primer momento, el marco teórico y los antecedentes, donde se recurre a los aportes investigativos de carácter nacional e internacional que ofrecen estudios diversos sobre el tema en cuestión. Además se presenta el contexto históricoeducativo de las escuelas normales superiores para introducir la presentación de la sistematización de las experiencias de maestros y maestras de las 11 escuelas, sobre el liderazgo educativo que están llamadas a ejercer en el siglo XXI y, de este modo, plantear la discusión a partir de las contrastaciones teórico-conceptuales y empíricas que implica. Se resalta que el liderazgo debe distinguirse por su capacidad de compenetrarse con el territorio, desde donde debe interactuar con las demás personas y su entorno. A la vez, que posee la capacidad para discernir las situaciones complejas y así asumir la postura más adecuada para promover la participación y la inclusión.

En un segundo momento, se desarrolla, de manera formal, la metodología, la cual se caracteriza por ser polifónica, en el sentido que se recurre a enfoques diferentes que guardan matizaciones diversas que han posibilitado la profundidad y el rigor en la investigación. Es una investigación descriptiva, con un enfoque metodológico cualitativo, sustentada en el método de investigación documental y técnicas de análisis documental que implican una metodología hermenéutica de los aportes recibidos desde las experiencias de las personas coordinadoras que asistieron.

\footnotetext{
${ }^{4}$ La metodología de innovación social educativa (ISE), según Acosta Valdeleón (2016), es un tipo de innovación educativa que recoge los aportes de la innovación social para producir respuestas creativas a problemas educativos. Comprende cinco pasos: 1. priorización (la comunidad elige un problema educativo para solucionarlo con la innovación), 2. caracterización (el problema es analizado para comprender cómo está compuesto y cómo funciona), 3. ideación (la comunidad educativa genera ideas disruptivas, las mejora y potencia), 4. prototipado (se pone a prueba la innovación, se ajusta y se mejora) y $\mathbf{5}$. adopción (la comunidad educativa adopta formalmente la innovación). El proceso de formación está pensado desde una metodología participativa que permite constituirse en comunidad de práctica innovadora en educación (COPIE) y emprender un proceso de innovación participativo que contribuya a la solución de los problemas educativos; por último, se busca incrementar la capacidad de agencia de las comunidades educativas.
} 
Por último, se presentan los hallazgos como resultado del análisis conceptual, comparativo y relacional de los aportes de las 11 personas líderes-coordinadoras de las escuelas normales superiores, que muestran cómo el liderazgo integral educativo innovador parte de la toma de consciencia de los problemas que emergen del territorio y de las instituciones educativas. Se prioriza la implementación de estrategias pedagógicas y la interacción del sentido pedagógico con la innovación y la creatividad. Y se asume, así, la coexistencia con el conflicto y el aprendizaje continuo. Tales aspectos llevan a identificar unos elementos particulares como: la pertenencia al territorio concebido como una unidad física y sociocultural que está en movimiento. Los proyectos educativos institucionales como escenarios vitales desde donde se pueden transformar los problemas en oportunidades con la participación de todos y todas, lo que lleva a una práctica axiológica que prioriza el diálogo, la escucha, la solidaridad y la confianza; y el fortalecimiento de competencias del orden de comprender aspectos teóricos y prácticos, participar en procesos comunitarios e incrementar el agenciamiento y el empoderamiento de las comunidades.

\section{Marco teórico: Antecedentes}

Como parte del estado de la cuestión, a continuación, se presentan los resultados de algunas investigaciones tanto nacionales como internacionales que se consideran relevantes y pertinentes al tema de interés. Luego se muestra el contexto histórico y educativo de las escuelas normales, al vincular las experiencias sistematizadas de 11 personas coordinadoras de estas escuelas que participaron en el Diplomado en Innovación Social Educativa (ISE), provenientes de diferentes regiones del país y que se constituyen en el valor agregado del presente artículo.

Los diferentes aportes sobre el liderazgo educativo priorizan la creatividad y las habilidades de las personas. Desde este enfoque, Sierra Villamil (2016) presenta su reflexión sobre el liderazgo educativo en el siglo XXI, a partir del emprendimiento sostenible. Describe el liderazgo educativo comenzando por los elementos clave que repercuten en el comportamiento de un sujeto líder desde una mirada de la sostenibilidad. Para la autora, el liderazgo educativo se evidencia en la creación de estrategias pedagógicas y la interacción del sentido pedagógico con la innovación y la creatividad, o en la mejora permanente de la práctica docente y la interacción del sentido pedagógico de sus líderes y la cohesión entre la visión del futuro de la organización teniendo presente el desarrollo de las personas. Igualmente enfatiza que el liderazgo tiene que ver con las personas y sus comportamientos.

Otro aporte significativo lo ofrecen Rodríguez Espinoza et al. (2012), desde Chile, con su investigación sobre el desarrollo del liderazgo educativo dentro del programa de liderazgo CRECE de la Pontificia Universidad Católica de Valparaíso. En este contexto, la labor investigadora consiste en narrar, relatar los hechos que acontecen en los sujetos como punto de partida para luego interpretar y así generar nuevas historias que registran transformaciones de enseñanzas y aprendizajes. Para tal fin, los autores siguen el método de corte cualitativo e interpretativo y que según Portela Pruaño et 
al. (2009), se ha desarrollado sobre la base de la formación de docentes. Un aspecto a resaltar de este trabajo es que concibe el liderazgo como una herramienta que integra las diferentes esferas de la experiencia vital. Además, enfatizan que es imposible separar el rol de líder de la persona líder.

Desde México, Vergara Alonso (2012) presenta su reflexión sobre el liderazgo en escuelas normales y reconoce cómo la categoría de liderazgo es un concepto que admite matizaciones de comprensión diversa y que, procesualmente, se ha constituido en un elemento central del discurso de las instituciones educativas. Al respecto, puntualiza que liderar en el contexto de la educación significa potenciar la habilidad para analizar y simplificar la información que circula en los medios y en el entorno, donde prolifera todo tipo de datos que carecen de referentes sólidos y verdaderos. Tal cometido debe ser tenido en cuenta para hablar de un liderazgo pertinente y actualizado, según lo exige el mundo de hoy. Además, se necesita reinventar nuevas formas de ejercer el liderazgo; esto implica poseer una perspectiva diferente que incluya la coexistencia de la tensión, del conflicto y el riesgo del aprendizaje continuo.

El punto nodal, según la autora, consiste en tomar conciencia de que liderar responsablemente la escuela normal implica un liderazgo que tenga visión y esté abierto al diálogo y a la negociación con los distintos sectores educativos que constituyen la sociedad. Por tanto, la discusión no debe centrarse en indagar significados para definir los estilos de liderazgos, sino que la reflexión y la práctica se deben orientar a revisar las estructuras de las escuelas y preguntarse qué tanto dichas estructuras posibilitan participación, autonomía, libertad para pensar y expresar lo que se siente, conscientes de los deseos de transformación.

Otra investigación igual de relevante es la que se orienta a explorar el liderazgo directivo, en donde Brito Lara et al. (2016) exponen hallazgos de una investigación encaminada a identificar las estrategias y características que pueden determinar exitoso el liderazgo directivo, específicamente en el ámbito de la gestión escolar de educación secundaria. Recurren a perspectivas contemporáneas sobre el liderazgo tales como: liderazgo carismático de Meléndez de León (1997) y Weber (2002), el liderazgo transformacional según Lupano Perugini y Castro Solano (2006) y el liderazgo estratégico de Lussier y Achua (2002). De estas comprensiones infieren la conceptualización del término y las características que lo pueden definir, al identificar cualidades personales y formas de gestión del personal directivo. Además, en el trabajo de campo estudiaron a personal directivo de escuelas secundarias del norte, centro y sur, y la participación de tres personas académicas de la Escuela Normal Superior de Guanajuato e identificaron que no es la preparación profesional de las direcciones lo que moviliza a la comunidad hacia los logros educativos, sino la habilidad de esta para armonizar las demandas internas y externas. Se trata de una implicación de las capacidades del sujeto directivo para generar empatía al interior de la institución educativa, sensibilidad para percibir y atender los hechos que se presentan a partir de las relaciones interpersonales neutras y exhortativas, potenciar los liderazgos de las demás personas y crear espacios de inclusión en la comunidad escolar en la que puedan participar el profesorado, alumnado, y padres y madres de familia. 
En la misma perspectiva, los aportes de Sánchez-Reyes y Barraza-Barraza (2015) presentan los resultados de su investigación sobre las percepciones ${ }^{5}$ del liderazgo en las instituciones formadoras y actualizadoras de docentes (IFAD). Encontraron que las funciones directivas estaban centradas en la administración escolar, aunque identificaron intentos por enfatizar los asuntos académicos, pues con frecuencia deben atender actividades que no tienen que ver con actividades escolares. Presentan los estilos de liderazgo a partir de diferentes autorías. Es una investigación que se ubica en el paradigma interpretativo, con un enfoque cualitativo y un método fenomenológico. La técnica fue la entrevista a profundidad y el instrumento guía que utilizaron fue la entrevista semiestructurada.

Por otra parte, el aporte notable de Gómez Ortiz (2006), en su ensayo sobre el liderazgo empresarial para la innovación tecnológica -si bien es cierto no corresponde al campo de la educación, pues presenta la innovación con su nicho en espacios empresariales- en cuyo escenario se muestra la creación de conocimiento innovador. Gómez Ortiz (2006) expone rigurosamente una visión panorámica sobre las teorías más relevantes alrededor del liderazgo, para inferir elementos básicos que permitan el desarrollo empresarial mexicano. Además, concluye que existen diversas concepciones sobre el liderazgo según la diversidad de quién escriba sobre ello. Señala, de forma descriptiva y analítica, los atributos asociados al liderazgo que se presentan de manera reiterativa, lo cual permite ver con mayor profundidad una tipología completa. A continuación, se transcriben, en la Tabla 1, los atributos asociados y, en la Tabla 2, los estilos de liderazgo y su descripción correspondiente.

De lo expuesto hasta el momento, se puede afirmar que el liderazgo es una realidad que acepta multiplicidad de comprensiones que están vinculadas al rol del líder y a la persona misma, la cual integra la experiencia vital; articula tensiones y conflictos a partir de una apertura al diálogo y la búsqueda de negociaciones; promueve pactos y consensos que se orientan hacia un bienestar colectivo; tiene en cuenta principios o normas naturales que lo regulen. Al respecto, Covey (2013) señala que el liderazgo debe regirse por principios básicos como la rectitud, la justicia, la equidad, la honestidad, la integridad y la confianza. Estos principios emergen de la urdimbre social y familiar, y estimulan el aprendizaje permanente como camino para afianzar la dimensión relacional de la condición humana en todos los ámbitos de la sociedad. Tales principios pueden fortalecer el liderazgo del profesorado a partir de su liderazgo pedagógico, como un medio para dar calidad a la educación. Tal como lo señala Bernal Martínez de Soria e Ibarrola García (2015):

${ }^{5}$ En esta investigación las personas autoras aclaran que la percepción está apoyada en la teoría Gestalt que tiene un enfoque holístico, porque percibe las cosas como totalidades, lo que significa que el todo es más que la suma de las partes. 
Tabla 1: Frecuencia de acciones atribuidas al liderazgo

\begin{tabular}{cl}
$\begin{array}{c}\text { N. }{ }^{\circ} \text { de } \\
\text { definición }\end{array}$ & Acciones de liderazgo de mayor frecuencia \\
\hline 1 & Senda, ruta, curso; curso, guía a las personas a su destino; unir a las personas en grupo. \\
2 & Influye, dirige, guía. Acciones de motivación al logro. Capacidad de decisión de las personas seguidoras. \\
3 & Ejerce influencia sobre otras personas, las dirige y guía hacia el logro de objetivos. \\
4 & Influye, guía y dirige hacia el logro de los objetivos y metas. \\
5 & Influye hacia el logro de metas. \\
6 & Influye en un grupo o en un individuo. \\
7 & Capacidad de decidir lo que debe hacerse. Motivar que las demás personas lo hagan. \\
8 & Influir en otros sujetos \\
9 & Hacen las cosas correctas. \\
10 & Motivación, acciones encaminadas a intereses de grupo. \\
11 & Inducir, comprometer y satisfacer los motivos de los sujetos seguidores. \\
12 & Acciones éticas y sociales para lograr los objetivos de los sujetos seguidores. \\
\hline &
\end{tabular}

Nota: Tomado de Gómez Ortiz (2006, p. 68).

Tabla 2: Estilos de liderazgo y sus descripciones

\begin{tabular}{|c|c|}
\hline Estilo & Descripción \\
\hline Egocéntrico & Centrado en sí, dominante, dirige el imperio desde el centro. \\
\hline Autocrático & Envía las respuestas desde la cima, no consulta. Es un ser impositivo. \\
\hline Carismático & $\begin{array}{l}\text { Posee una fuerte personalidad que utiliza para lograr que las personas hagan las } \\
\text { cosas como dice. }\end{array}$ \\
\hline $\begin{array}{l}\text { Igualitario superior, } \\
\text { primero entre los iguales }\end{array}$ & $\begin{array}{l}\text { Persona participativa, pero siempre da la impresión de conocer la respuesta, hace } \\
\text { que las personas se sientan superiores y dignas de confianza. }\end{array}$ \\
\hline Inteligencia superior & Estos sujetos líderes intimidan a las personas sin darse cuenta. \\
\hline Conflicto & Utiliza el conflicto, la ira y las pugnas para inspirar y presionar a las personas hacia el éxito. \\
\hline Constructor de equipos & $\begin{array}{l}\text { Mima y desarrolla a los equipos hasta que alcanzan resultados superiores por el solo } \\
\text { hecho de querer agradar a su líder. }\end{array}$ \\
\hline
\end{tabular}

continúa 


\begin{tabular}{ll}
\hline Estilo & Descripción \\
\hline Estratégico & $\begin{array}{l}\text { Comunica siempre la visión y la trayectoria hacia delante, enfocada y no complicada, } \\
\text { se le respeta por la claridad de la imagen social. }\end{array}$ \\
\hline Popular & $\begin{array}{l}\text { Persona extraordinaria que se presenta en apariencia ordinaria, aunque hace que } \\
\text { quienes le rodean se sientan sujetos extraordinarios. }\end{array}$ \\
\hline Pastor & Trata a su personal, clientela y demás participantes con cuidado y solicitud. \\
& Tiende a empujar en lugar de jalar. Comprende muy bien a los demás individuos. \\
\hline General del ejército & $\begin{array}{l}\text { Trata de poner grandes ejemplos, pero espera que su personal siga sus órdenes sin } \\
\text { cuestionarlas. }\end{array}$ \\
\hline Líder de realeza & $\begin{array}{l}\text { Se percibe como aristócrata natural de liderazgo, sabe hacer lo adecuado y cuándo } \\
\text { hacerlo. }\end{array}$ \\
\hline Político & $\begin{array}{l}\text { Siempre equilibra y manipula a los grupos participantes para mantener los nexos de } \\
\text { poder equilibrados y controlados en el centro. }\end{array}$ \\
\hline Líder natural & $\begin{array}{l}\text { Siempre luce cómodamente en una posición de liderazgo. Se conduce en la función } \\
\text { como si fuera lo único que hubiera hecho. Hace que los sujetos líderes nominales } \\
\text { sean tan inadecuados como de hecho, con frecuencia, lo son. Se les envidia lo } \\
\text { natural de sus dones y cualidades de liderazgo. }\end{array}$ \\
\hline
\end{tabular}

Nota: Tomado de Gómez Ortiz (2006, p. 80).

El impulso del liderazgo del profesor constituiría una de las metas principales de la gestión en centros educativos si se aspira al liderazgo pedagógico. Centrarnos en el liderazgo del profesor nos facilitaría encontrar más evidencias de la relación entre el liderazgo pedagógico y los resultados escolares; al fin y al cabo, lo que sí se ha podido valorar mejor es la relación entre docencia y aprendizaje ... teniendo en cuenta los contextos culturales y sociales concretos. ... La pista fundamental para entrever este interés en prácticamente todos los estudios que hemos revisado es encomendar a los gestores escolares la urgente tarea de promover el profesionalismo docente. El desarrollo profesional de un docente supondría dos líneas de actividad: trabajo en el aula y trabajo como líder, mientras que los aspectos en los que debiera formarse son: conocimientos disciplinares y su correspondiente didáctica, conocimiento pedagógico, conocimiento de las relaciones con los demás agentes educativos, así como del contexto y la actualización de la identidad profesional -misión, sentido de la enseñanza, cultura educativa, sentido de la comunidad y la organización educativas-. (p. 67)

En esta perspectiva, el maestro o la maestra, como líder, debe atender la realidad interna (aula) y la externa (entorno). Esta articulación se evidencia en el acompañamiento a las 11 escuelas normales superiores que, desde la óptica histórica, surgen como desafío ante la situación que vive Colombia por construir la paz. Aquí, la educación rural es un elemento clave para impulsar 
los diversos procesos de desarrollo y las estrategias que garanticen la construcción de escenarios de paz, con un manejo adecuado de las distintas situaciones de conflicto. En este contexto, la Universidad de la Salle crea el Laboratorio de Innovación Educativa en Formación de Maestros y Maestras Rurales como un escenario de investigación, intervención e innovación educativa que busca construir y proponer alternativas educativas, pedagógicas y didácticas que se orienten a mejorar los procesos de formación de los educadores y las educadoras rurales que necesita el país.

En este marco se implementan acciones para fortalecer los Programas de formación complementaria de 11 escuelas normales superiores de Colombia, seleccionadas mediante la convocatoria Acompañamiento Programas de Formación Complementaria en el fortalecimiento de la innovación en los procesos de formación de maestros y maestras rurales.

Las escuelas normales superiores han marcado la historia nacional y regional por una tradición de preservar la cultura pedagógica a través de la formación de personas dedicadas a la educación y atendiendo las situaciones de precariedad del territorio. Son instituciones formadoras de maestros y maestras para la educación preescolar y básica primaria. Según la ley 115 de febrero 8 de 1994 en el artículo 112 (Congreso de la República, 1994):

Las escuelas normales debidamente reestructuradas y aprobadas, están autorizadas para formar educadores en el nivel de preescolar y en el ciclo de educación básica primaria. Estas operarán como unidades de apoyo académico para la formación inicial de docentes y, mediante convenio celebrado con instituciones de educación superior, podrán ofrecer formación complementaria que conduzca al otorgamiento del título de normalista superior. (p. 24)

Así, dichas escuelas son las primeras instituciones acreditadas en el país para formar maestros y maestras. Muchas de ellas se encuentran en zonas de conflicto y en los cascos urbanos de los municipios. De ahí que se constituyen en un escenario vital para el desarrollo de las regiones a partir de la educación. Por tal motivo, es de suma importancia prestar atención a algunas experiencias sistematizadas, las cuales se entienden como "proceso de reflexión e interpretación crítica sobre la práctica y desde la práctica, que se realiza con base en la reconstrucción y ordenamiento de los factores objetivos que han intervenido en esa experiencia, para extraer aprendizajes y compartirlos" (Cifuentes Gil, 2017, pp. 11-12). Las 11 personas coordinadoras de las escuelas normales superiores que participaron en el Diplomado en Innovación Social Educativa (ISE), provienen de diferentes regiones del país, tales como Barrancabermeja, Mojana, Pitalito, Nocaima, Montes de María, Rio de Oro, Putumayo, Pupiales, El valle, Meta y Roldanillo. Cabe mencionar que las acciones que se implementaron durante todo el diplomado fueron de organización, participación, capacitación, planificación e innovación. Los objetivos de formación que se persiguieron se recogen en la Tabla 3. 
Tabla 3: Objetivos de formación del diplomado ISE

\begin{tabular}{ll}
\hline Caracterizar & $\begin{array}{l}\text { Las necesidades y potencialidades de las experiencias de innovación en relación con la investigación } \\
\text { educativa, construcción de paz, convivencia y valores en las } 11 \text { escuelas normales superiores. }\end{array}$ \\
\hline Analizar & $\begin{array}{l}\text { Conjuntamente elementos teóricos y metodológicos de la innovación social educativa como } \\
\text { alternativa de fortalecimiento de experiencias de innovación en relación con la investigación } \\
\text { educativa y la construcción de paz, convivencia y valores en la formación de docentes. }\end{array}$ \\
\hline Sistematizar & $\begin{array}{l}\text { Los procesos de innovación social educativa liderados por los entes coordinadores del } \\
\text { Programa de Formación Complementaria en torno a la investigación educativa y la } \\
\text { construcción de paz, convivencia y valores en la formación de docentes. }\end{array}$ \\
\hline Incrementar & $\begin{array}{l}\text { La capacidad de agencia y liderazgo de los entes coordinadores de Programa de Formación } \\
\text { Complementaria en sus comunidades educativas y su entorno social. }\end{array}$ \\
\hline Promover & $\begin{array}{l}\text { La sostenibilidad y transferencia de los procesos de innovación en investigación educativa y la } \\
\text { construcción de paz, convivencia y valores en la formación de docentes. }\end{array}$ \\
\hline
\end{tabular}

Nota: Elaboración propia.

El diplomado se desarrolló en cinco sesiones de tres días cada una y se complementaron con encuentros virtuales durante el II semestre 2017 y I semestre de 2018. El desarrollo metodológico articuló aspectos temáticos, trabajos previos, actividades y evaluación, como lo muestra la Figura 1.

Figura 1: Desarrollo metodológico del diplomado ISE

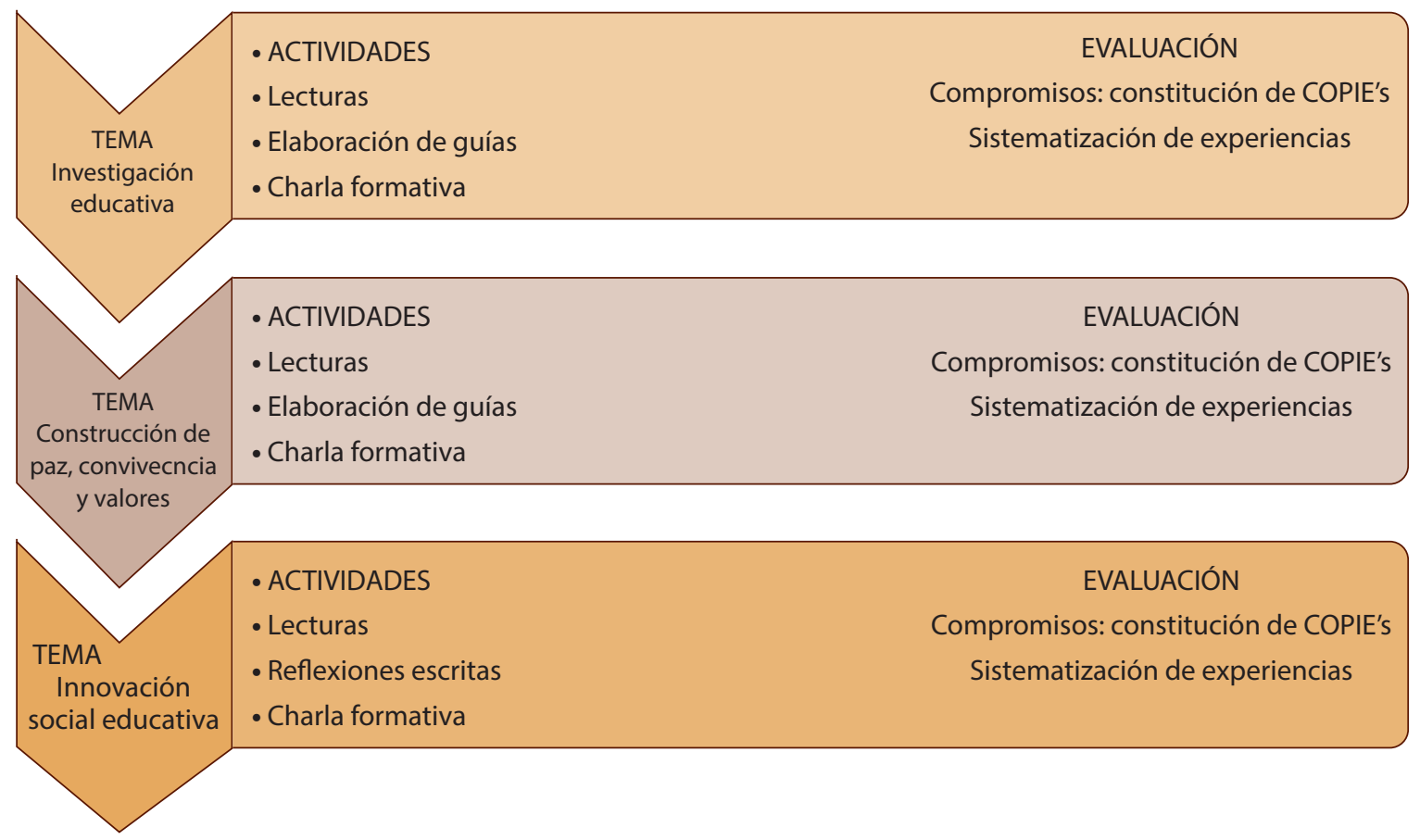

Nota: Elaboración propia.

10 Amparo Novoa-Palacios

Los artículos de la Revista Electrónica Educare del Centro de Investigación y Docencia en Educación de la Universidad Nacional, Costa Rica, se comparten bajo términos de la Licencia Creative Commons: Reconocimiento, № Comercial, Sin Obra Derivada 3.0 Costa Rica. Las autorizaciones adicionales a las aquí delimitadas se pueden obtener en el correo: educare@una.cr 
La experiencia de sistematizar es sobre el rol del líder que se abordó en el tema construcción de paz; pero más específicamente en convivencia y valores que estuvo a cargo de la autora del presente artículo. Se aplicaron talleres en las distintas sesiones sobre valores y en la última se impartió una charla con el tema el liderazgo y la innovación, la cual se centró en la comprensión del ser líder en el contexto educativo y la necesidad de impartir una formación integral con un carácter innovador. De aquí se infirió que el primer cometido del liderazgo es pensar, elaborar y guiar con el ejemplo a los demás sujetos con respecto a los fines, valores y objetivos compartidos. Esto se visibiliza a partir de una estructura organizacional que debe crearse sobre la base de la comunicación, y la responsabilidad personal y social; que implica la responsabilidad moral de quien lidera, que debe tener una firme convicción para alcanzar eficacia en los procesos.

La charla fue complementada con dos talleres como fuente de información, con preguntas de carácter personal y reflexivo que indagaban sobre los valores identificados en sus instituciones y en las comunidades de práctica innovadoras en educación (COPIE's) y sobre sus actitudes específicas como líderes de sus instituciones con el rol de coordinación. Tales preguntas las trabajaron individual y colectivamente, y se formularon para explorar la capacidad mostrada para comprender a los demás seres. Son preguntas que se orientan al autoconocimiento. Con la intención de comprender mejor este aspecto, Gardner (1993) describe ocho clases de inteligencias, entre las cuales se encuentran la inteligencia interpersonal y la intrapersonal. La primera se centra en las personas que hacen contacto permanente con otras, en este caso: el personal docente, y la segunda son las personas que poseen un conocimiento de sí mismas y aplican sus cualidades de forma exitosa hacia las demás. Para Gardner (1997), toda persona posee este tipo de inteligencias, ya que todas las funciones sociales necesitan más de una inteligencia. Sin embargo, no se puede desconocer que las investigaciones posteriores sobre las inteligencias han cuestionado si el aporte de Gardner es realmente una teoría científica, o simplemente es una reformulación de estilos cognitivos que, en su momento, estaban de moda (Larivée, 2010). Sea lo que sea, es importante anotar que estas dos inteligencias son fundamentales para potenciar un liderazgo que abogue por el bien colectivo $y$, en la actualidad, urge fomentar dichos aspectos en el liderazgo.

A continuación se presenta, en la Tabla 4, la sistematización sobre los valores y, en la Tabla 5, la sistematización sobre actitudes. De aquí será posible develar rasgos para elaborar un perfil sobre el liderazgo integral educativo desde una visión axiológica e innovadora, para luego contrastar con las fuentes teóricas expuestas e identificar la novedad. 
http://doi.org/10.15359/ree.24-3.2

http://www.una.ac.cr/educare

educare@una.ac.cr

Tabla 4: Sistematización sobre valores

1. ¿Qué valores ve necesarios que el país debe priorizar en la época del pos-acuerdo?

- El sentido de pertinencia, el reconocimiento y aceptación del otro ser (diversidad) y la toma de conciencia.

- El sentido de superación con educación incluyente. Equidad en la distribución de la riqueza nacional. Desarrollo humano con justicia social. La resiliencia y el trabajo colectivo.

- Reconciliación basada en el perdón, la honestidad para el uso de los recursos comunes, el crecimiento espiritual fuente de inclusión.

- La tolerancia, la igualdad de oportunidades y el perdón.

- Diálogo respetuoso y justicia social.

2. ¿Qué valores identifico en mi institución como los más fuertes?

- El pluralismo, pues se fomentan variadas formas de pensamiento que hacen parte de la dinámica escolar y cultural.

- Sensibilidad artística, respeto por la diferencia, responsabilidad docente, buen trato, disponibilidad para emprender tareas, orgullo por la escuela.

- La responsabilidad como eje del trabajo y del estudio y la solidaridad.

- El servicio, la solidaridad, la generosidad, la responsabilidad social.

- Respeto por la diferencia y la solidaridad.

- Transparencia en el manejo de los recursos.

- La interculturalidad y la autonomía.

3. ¿Qué valores se requieren fortalecer en mi institución?

- El sentido de pertenencia. Es necesario que el colectivo docente se apropie de los lineamientos y dinámicas de la institución y del contexto para ser más proactiva y enriquecedora su labor.

- Trabajo en equipo, apertura a cambios estructurales, transparencia en el manejo de lo financiero, solidaridad, mayor sentido a la profesión docente, mejor comunicación con la totalidad de integrantes de la comunidad educativa.

- El vivir como comunidad de aprendizaje.

- El sentido de pertenencia, el compromiso y dedicación de cada integrante de la comunidad educativa, falta empatía.

- Trabajo en equipo y la comunicación.

- La humildad.

- El liderazgo asertivo y estratégico.

- El trabajo investigativo y la tolerancia en situaciones difíciles.

4. ¿Qué valores son fundamentales promover para dinamizar una comunidad de práctica innovadora en educación, COPIE?

- Fortalecer la comunicación asertiva, liderazgo, trabajo en equipo y apertura al cambio.

- Trabajo en equipo, liderazgo, sentido de pertenencia, pasión por lo que se hace y visión hacia el bien común.

- Sentido de la comunidad, el diálogo, la diferencia como elemento de crecimiento, organización, celebrar y comunicar.

- La motivación, la paciencia, la perseverancia, la tolerancia a la frustración.

- Alegría, responsabilidad y empatía.

- Disponibilidad, voluntad, respeto por las ideas de otros sujetos.

- Participación, comunicación, respeto y sentido de pertenencia.

Nota: Elaboración propia.

12 Amparo Novoa-Palacios

Los artículos de la Revista Electrónica Educare del Centro de Investigación y Docencia en Educación de la Universidad Nacional, Costa Rica, se comparten bajo términos de la Licencia Creative Commons: Reconocimiento, № Comercial, Sin Obra Derivada 3.0 Costa Rica. Las autorizaciones adicionales a las aquí delimitadas se pueden obtener en el correo: educare@una.c 
http://doi.org/10.15359/ree.24-3.2

http://www.una.ac.cr/educare educare@una.ac.cr

Tabla 5: Sistematización sobre actitudes

\begin{tabular}{|c|c|c|c|}
\hline Pregunta & Dimensión personal & Dimensión institucional & Dimensión educativo-social \\
\hline ¿Qué me motiva? & $\begin{array}{l}\text { - Ayudar a cumplir con compromisos } \\
\text { sociales con los diferentes municipios. } \\
\text { - Ayudar a crear mundos campesinos } \\
\text { posibles. Esto es una apuesta } \\
\text { fundamental. } \\
\text { - Saber que hay vocación de servicio. } \\
\text { - Identificar talento en lo personal y } \\
\text { colectivo. } \\
\text { - La necesidad de cualificación para } \\
\text { seguir sirviendo en una actitud de } \\
\text { agradecimiento y servicio recíproco. } \\
\text { - La motivación de tener que seguir } \\
\text { aprendiendo. Ser parte de la solución } \\
\text { y un compromiso de la formación } \\
\text { docente. } \\
\text { - La transformación acontece en la } \\
\text { transformación del maestro o maestra, } \\
\text { por ello hay que involucrar a toda la } \\
\text { comunidad. } \\
\text { - Saber que lo que hago es válido. }\end{array}$ & $\begin{array}{l}\text { - Afirman que el sentido de } \\
\text { pertenencia a la institución } \\
\text { educativa genera actitudes } \\
\text { proactivas y confluencia de } \\
\text { proyectos. Esto empieza con } \\
\text { cada quien, en el ser capaz de } \\
\text { administrarse. } \\
\text { - La necesidad de cambiar } \\
\text { paradigmas administrativos. } \\
\text { - Hacer de la comunidad } \\
\text { educativa una institución } \\
\text { reconocida en la formación de } \\
\text { valores. } \\
\text { - Los motiva contribuir al } \\
\text { desarrollo educativo de sus } \\
\text { instituciones. } \\
\text { - La transformación acontece en } \\
\text { la transformación del maestro } \\
\text { o maestra, por ello hay que } \\
\text { involucrar a toda la comunidad. }\end{array}$ & $\begin{array}{l}\text { - Motiva a revisar los aprendizajes } \\
\text { de estudiantes y profesorado para } \\
\text { que no se orienten por la repetición } \\
\text { y sí por forjar sueños y hacerlos } \\
\text { posibles, pues la educación es una } \\
\text { herramienta transformadora que } \\
\text { debe potenciar las capacidades } \\
\text { desde una formación integral para el } \\
\text { cambio (tanto del profesorado como } \\
\text { de estudiantes). } \\
\text { - Implementar procesos de } \\
\text { innovación, que permitan la } \\
\text { relectura desde las prácticas para } \\
\text { identificar dónde se está estancado } \\
\text { y poder llevar lo teórico a la práctica. } \\
\text { - La educación es un asidero posible. } \\
\text { - La transformación acontece en la } \\
\text { transformación de cada docente, } \\
\text { por ello hay que involucrar a toda la } \\
\text { comunidad. }\end{array}$ \\
\hline $\begin{array}{l}\text { ¿Qué me anima y } \\
\text { me fortalece? }\end{array}$ & $\begin{array}{l}\text { - Convertirme en un referente para mis } \\
\text { - El amodiantes. } \\
\text { - Poder compartir, transferir la } \\
\text { experiencia, no sólo replicar, es mucho } \\
\text { más que eso. } \\
\text { - La curiosidad de saber claramente } \\
\text { cómo innovar. } \\
\text { - Me ayuda poder contribuir con } \\
\text { proyectos de vida para la niñez } \\
\text { campesina. } \\
\text { - A pesar de las discordias con el rector, } \\
\text { de alguna manera se me reconoce el } \\
\text { trabajo rural. Es un reconocimiento al } \\
\text { trabajo y esto es lo importante. } \\
\text { - El interés por aprender cosas nuevas y } \\
\text { aprende de los errores. } \\
\text { - Me anima la receptividad del } \\
\text { estudiantado. } \\
\text { - Ser insistentes en persistir, resistir. } \\
\text { - El trabajo en equipo, compartir sueños } \\
\text { e ideales. } \\
\text { - Contar con amigos y amigas de camino. } \\
\text { - Cuidar el ejercicio del poder. } \\
\text { - Ser parte de las comunidades con } \\
\text { quienes vivo la experiencia. } \\
\text { - Vivir para poder transformar para que } \\
\text { se vuelva más poderoso. }\end{array}$ & $\begin{array}{l}\text { - Los cambios que se producen en } \\
\text { las instituciones donde estamos. } \\
\text { - Que hay equipo, el entusiasmo } \\
\text { - El compartido. } \\
\text { institución. Por ejemplo, el } \\
\text { profesor de informática, la } \\
\text { profesora de práctica y las } \\
\text { profesoras de ética. } \\
\text { - La formación que se nos brinda } \\
\text { por parte de otras instituciones, } \\
\text { nos ayuda a formar. } \\
\text { - La escuela normal tiene } \\
\text { reconocimiento en la región, } \\
\text { me anima a seguir insistiendo. } \\
\text { Haciendo proyectos. } \\
\text { - La credibilidad que hoy tiene } \\
\text { la escuela normal superior. } \\
\text { Se valora los procesos de } \\
\text { capacitación. }\end{array}$ & $\begin{array}{l}\text { - Creer que se puede transformar } \\
\text { desde la educación la aldea global. } \\
\text { - La escuela normal tiene } \\
\text { reconocimiento en la región, me } \\
\text { anima a seguir insistiendo. Haciendo } \\
\text { proyectos. } \\
\text { - Lo rural es un compromiso de todos. } \\
\text { - En la COPIE, Ilevamos al contexto } \\
\text { las prácticas de docentes y } \\
\text { específicamente la innovación. } \\
\text { - La innovación social hace ver las } \\
\text { injusticias. } \\
\text { - Implementar proyectos, por } \\
\text { ejemplo: se inició un proceso } \\
\text { llamado el plan padrino para niños y } \\
\text { niñas que encuentran barreras. }\end{array}$ \\
\hline
\end{tabular}

continúa 


\begin{tabular}{|c|c|c|c|}
\hline Pregunta & Dimensión personal & Dimensión institucional & Dimensión educativo-social \\
\hline $\begin{array}{l}\text { ¿Qué me hace } \\
\text { dudar y me } \\
\text { obstaculiza? }\end{array}$ & 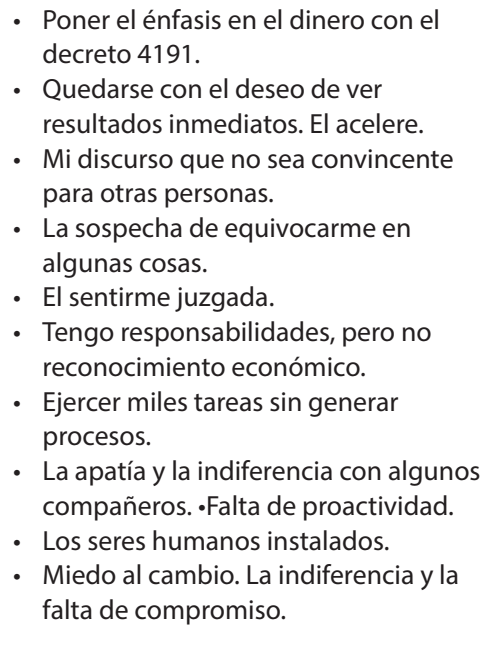 & $\begin{array}{l}\text { - Los cambios institucionales no } \\
\text { permiten procesos. } \\
\text { - El despido de personal. } \\
\text { - Personas que no participan } \\
\text { ejercen una crítica destructiva. } \\
\text { - La resistencia al cambio de } \\
\text { algunos miembros de la } \\
\text { comunidad. Están en la zona de } \\
\text { confort. } \\
\text { - El estatuto donde los profesores } \\
\text { están, se sienten perfectos, que } \\
\text { siempre hacen lo correcto. } \\
\text { - Pensarnos como los dueños de } \\
\text { la verdad en la institución. } \\
\text { - Profesores que siente miedo a } \\
\text { equivocarse. } \\
\text { - La falta de recurso económico } \\
\text { para implementar lo nuevo. } \\
\text { El poco tiempo institucional. }\end{array}$ & $\begin{array}{l}\text { - Las políticas del gobierno y el } \\
\text { Estado. } \\
\text { - Las realidades del entorno } \\
\text { educativo. } \\
\text { - No simplemente se aprende en un } \\
\text { salón con una temática sino que } \\
\text { existe en el contexto algo más. }\end{array}$ \\
\hline
\end{tabular}

Nota: Elaboración propia.

\section{Discusión}

Las experiencias sistematizadas en las investigaciones sobre el liderazgo de las distintas instituciones referenciadas ponen de relieve la concepción que se tiene sobre el liderazgo en los contextos educativos rurales. A la vez, muestran procesos y estrategias que favorecen la revisión del líder y su disposición al cambio para potenciar un perfil de líder innovador. Resaltadas en las mismas acciones, voluntades y decisiones que han sido expresadas para alcanzar el objetivo.

Las experiencias muestran la importancia de articular las disposiciones actitudinales que implican la inteligencia inter e intra personal, las cuales priorizan una serie de valores en orden al contexto del país y a la comunidad educativa inserta en él. Para este fin, las experiencias de las personas coordinadoras de las escuelas normales superiores insisten en potenciar valores que permitan la generación de sentido, de pertenencia al país y a la institución, lo que muestra el camino para amortiguar el individualismo y la llamada al Estado para que preste mayor atención a los contextos rurales. Con este fin, el Ministerio de Educación Nacional (2012) elaboró un manual para la formulación y ejecución de planes de educación rural con el objetivo de "hacer visible e incluir en el desarrollo nacional a la gente que vive y produce en la zona rural, sin cuya producción y vitalidad, las zonas predominantemente urbanas no podrían desarrollarse" (p. 9).

Reflexionar sobre la pertenencia como valor implica afianzar la inclusión y la cohesión social en medio de una sociedad sedienta de paz con justicia social y equidad con igualdad, que en últimas es dar sentido a la vida en todas sus dimensiones. De ahí la importancia de tratar la pertenencia 
como un valor que debe ser articulado a un concepto inclusivo de ciudadanía, asunto que aborda Prats Gil (2007) como una tarea urgente que deben asumir todos los sistemas educativos.

Alcanzar esa ciudadanía inclusiva, es imprescindible que antes se desarrolle una noción identitaria no basada en discriminaciones y alejamientos, sino en proximidades y acercamientos. En esta noción acogedora de la identidad, la idea de igualdad adquiere un alto potencial pedagógico; de hecho, la educación ha tenido, en la igualación, una de sus finalidades tradicionales, como un rasero que elimina y pule las impurezas. (p. 122)

De este modo, la pertenencia se da a través del reconocimiento y aceptación del otro, la tolerancia y la igualdad de oportunidades, en cuyo escenario el concepto de ciudadanía debe ser "capaz de motivar a los miembros de una sociedad a prestar su adhesión a proyectos comunes sin emplear para ello recursos embaucadores, exige enfrentar un conjunto amplio de problemas, heredados a menudo, y nuevos en ocasiones" (Cortina, 2009, p. 30). De este modo, la pertenencia como valor educativo y ciudadano no puede surgir como imposición, sino como toma de conciencia de que somos seres humanos y semejantes.

Habitar un mundo en el que las cosas no sólo son lo que son sino lo que también significan; pero lo más humano de todo es comprender que, si bien lo que sea la realidad no depende de nosotros, lo que la realidad significa sí resulta competencia, problema y en cierta medida opción nuestra. (Savater, 1991, p. 31)

Por otra parte, las personas coordinadoras de las ENS resaltan que el desarrollo humano con justicia social y honestidad sean los valores que sostengan el contexto de reconciliación y perdón. Asimismo, identifican valores tales como: pluralismo, responsabilidad, servicio, solidaridad, transparencia, autonomía, disponibilidad, trabajo en equipo, entre otros que fortalecen la institución y deben cuidar aquellos valores que están menos evidentes como: el sentido de pertenencia; el vivir como comunidad de aprendizaje; el trabajo en equipo; la comunicación clara; el liderazgo asertivo y estratégico; la apertura a cambios estructurales como camino para superar organizaciones burocráticas, verticales y rígidas. Sobre este asunto, Vergara Alonso (2012), en su investigación, afirmó que se necesitan nuevas formas de ejercer el liderazgo, donde se prioricen perfiles que posean perspectiva, visión y, sobre todo, deseo de aprender. Igualmente, Bolívar (2010) defiende su tesis de que, para mejorar la educación, es necesario cambiar los modelos de dirección de las instituciones educativas, pues el modelo actual administrativo-burocrático presenta déficits.

Tales valores pueden ser potencializados a partir de las comunidades de práctica innovadora en educación (COPIE's) que ya están constituidas en cada escuela y necesitan el ejercicio de un liderazgo que priorice la apertura al cambio, la comunicación, el trabajo en 
equipo, el diálogo, la motivación, la responsabilidad como aspectos axiológicos que posibilitan el crecimiento y la organización de la comunidad educativa hacia la innovación con un liderazgo estratégico y asertivo.

Las experiencias sistematizadas sobre actitudes revelan lo que hay en la esfera intrapersonal y donde se articulan las dimensiones personal, institucional y educativa-social. Es una triada que muestra el rostro concreto de la experiencia de las personas coordinadoraslíderes. Y en ella se refleja que las motivaciones que cada una identificó, les mueve a ser parte de la solución, lo que muestra rasgos de un liderazgo asertivo y estratégico, que está en camino al cambio. Es importante hacer mención de que la actitud se comprende, según la Real Academia Española (2018), como "disposición de ánimo manifestada de algún modo. ... Postura del cuerpo especialmente cuando expresa un estado de ánimo" (párr. 1-2). Sin las actitudes sería difícil el aprendizaje y el crecimiento personal, por ello es fundamental que los maestros y las maestras adviertan "cuál es el significado que cabe otorgar esencialmente a las actitudes y cómo se relacionan los diferentes aspectos conductuales, cognitivos y afectivos que en ellas convergen" (Asensio Aguilera, 2002, p. 52). En la sistematización de experiencias las disposiciones actitudinales son relevantes en cuanto que permiten contemplar el rostro del otro desde valores de apertura y acogida, "la relación con el otro no es una relación contractual o negociada, no es una relación de dominación ni de poder, sino de acogimiento. Es una relación ética basada en una nueva idea de responsabilidad" (Bárcena y Mèlich, 2000, p. 15). De esta manera, los valores se constituyen en dinamizadores de las conductas particulares y se constituyen en un componente vital para el liderazgo innovador.

Las personas coordinadoras también conciben su vocación de maestros y maestras como una vocación de servicio y entrega incondicional en la que reconocen que la transformación acontece en la transformación de su propio ser.

El rasgo más importante para distinguir a un líder es su capacidad de conocer con profundidad (insight) la realidad, la interacción humana y el entorno de su escuela y, derivado de este insight, que sea capaz de discernir cuál es o debiera ser su conducta, estilo, tipo y actitud como líder. (Andere, 2017, p. 27)

De esta forma, se puede crear otra educación posible, cuya apuesta fundamental implica la revisión de los aprendizajes tanto de estudiantes como de docentes para que no sean experiencias repetitivas y se orienten a forjar sueños posibles.

Al constatar la educación como una mediación transformadora que debe potenciar las capacidades de los seres humanos desde una formación integral para el cambio, es prioritario implementar procesos de innovación que reconozcan la relectura desde las prácticas como camino de articulación de lo teórico y lo práctico. De este modo, la escuela normal superior 
podrá tener reconocimiento en la región y continuar insistiendo en un diálogo abierto con otras instituciones del contexto, situación que permitirá salir del estado de confort en que se encuentran algunos maestros y maestras $y$, por ende, la institución, lo cual se manifiesta en la resistencia al cambio. Por ello, es relevante saber que las realidades del entorno educativo pueden ser escenarios de aprendizaje.

\section{Metodología}

La metodología que se ha empleado responde a una investigación descriptiva que tiene presente los procesos prácticos de las escuelas normales superiores socializados por las personas coordinadoras que asistieron al Diplomado. Estableciendo una relación tripartita entre los factores que aluden al fenómeno multidimensional de orden interno y externo de las escuelas, las personas actoras en nuestro caso, la población representada en las 11 personas coordinadoras de las escuelas y las variables, aspectos que, en nuestro caso, se recogen en el enfoque metodológico cualitativo que reúne las características fundamentales sobre el liderazgo en el contexto educativo. Todo lo mencionado se sustenta en el método de investigación documental que, como técnica de recolección de datos, permitió usar diferentes tipos de documentos y escritos de las personas coordinadoras que participaron en el Diplomado y que han posibilitado reflexionar sistemáticamente sobre las realidades existenciales y teóricas contempladas en la investigación. En este sentido, también implica una metodología hermenéutica, pues ha supuesto un ejercicio de comprensión, diálogo y empatía para llegar a reconocer las experiencias de las personas coordinadoras como escenarios vitales que generan sentido a través del liderazgo que ejercen. Los resultados del análisis conceptual, comparativo y relacional de las contribuciones de las 11 personas líderes-coordinadoras de las escuelas normales superiores muestran la necesidad de un abordaje integral del liderazgo educativo rural en Colombia

\section{Reflexiones finales}

Los elementos mencionados podrían ayudar a configurar un perfil sobre el liderazgo integral educativo innovador que contribuya a la solución de los problemas socioeducativos con respuestas situadas en el territorio rural y así superar creativamente los factores adversos que la realidad impone. Para ello, es necesario que el liderazgo educativo evidencie la creación de estrategias pedagógicas y la interacción del sentido pedagógico con la innovación y la creatividad para integrar las diferentes esferas de la experiencia vital. Además, el liderazgo debe estar abierto al diálogo, la negociación, y a asumir la coexistencia de la tensión, el conflicto y el aprendizaje continuo. No se puede perder de perspectiva que el rol de líder está vinculado a la persona y todos sus atributos deben ser expresados en acciones concretas que prioricen la 
creación de espacios de inclusión y mayor participación. Para tal fin, se puede señalar algunos elementos para la construcción de un liderazgo integral educativo que asuma un marco de valores que realmente lo hagan innovador. Tales elementos se organizan en rasgos esenciales que deben tener en cuenta:

1. Los territorios. Lugares donde las escuelas normales superiores están asentadas; pero, además de ello, donde acontecen las diversas situaciones de trabajo, política, cultura que viven los individuos y las comunidades para configurar su vida colectiva. De ahí que el territorio no se pueda comprender sólo como espacio geográfico, sino que debe comprenderse como una unidad física y social en la cual interactúan instituciones diferentes con toda la población. Además, se da en lo rural que hace que la identidad territorial se defina por unas características muy particulares y que hoy se orientan a desmontar todos los prejuicios y estereotipos que vinculan lo rural a espacios desprovistos de progreso y crecimiento social. Según Bustos Jiménez (2006):

Sus habitantes forman parte de un colectivo social con códigos culturales concretos, léxicos y símbolos característicos, costumbres diferentes y comportamientos basados en las interacciones multifacéticas de sus miembros. Existe una... ciudadanía... a través de la cual el individuo se reconoce... como parte integrante de la comunidad. (p. 65)

Por tanto, se infiere que son poblaciones que hoy van tomando mayor protagonismo y se instituyen, en algunos países como Colombia, en escenarios en donde se debe invertir para el desarrollo de la sociedad.

2. Los proyectos educativos institucionales (PEI). Los aspectos normativos determinan pautas y secuencias operativas que deben desarrollar para hacer realidad el liderazgo que se necesita a partir de los valores identificados en la institución y los que precisan ser fortalecidos.

Los territorios donde están inmersas las escuelas normales son diferentes en sus necesidades y fortalezas, de ahí que sea importante saber cuáles valores son los prioritarios de vivir y potenciar para aportar a la solución de las problemáticas concretas. El liderazgo integral educativo innovador deberá tener la capacidad de transformar el problema como oportunidad y camino real de solución, por ejemplo, si en el contexto es fuerte el conflicto, la no escucha, será urgente implementar estrategias de diálogo que generen conciencia para hacer una comunidad participativa y dar el lugar a la palabra emitida por los grupos integrantes de la comunidad. En este sentido, Vergara Alonso (2012) afirma que el liderazgo implica tener visión y apertura al diálogo y así negociar con los distintos sectores educativos y sociales que forman parte del entorno. O en el caso de un contexto de desconfianza, será primordial liderar procesos que permitan creer en las personas reconociéndoles sus esfuerzos y aportes. Es así como la persona líder deberá tener su mirada puesta en favorecer la participación de cada integrante de la comunidad, lo cual pasa por el reconocimiento. 
El líder integral innovador, en su plano interpersonal, debe tener claro que la razón de su rol está en facilitar, permanentemente, el interactuar de todos los grupos integrantes de la comunidad educativa, donde prima los intereses colectivos por encima de los individuales, lo cual supone un conocimiento intrapersonal de su liderazgo.

3. Fortalecimiento de las competencias. Este aspecto se considera como un denominador común en el liderazgo integral educativo innovador, lo cual implica: 1. Comprender elementos teóricos y prácticos o, como bien lo señalan las personas coordinadoras-líderes de las escuelas normales: potenciar la cualificación para seguir sirviendo recíprocamente y con una actitud de agradecimiento. 2. Participar de manera crítica y propositiva en las COPIE, se trata de ser parte de la solución. 3. Incrementar la capacidad de agenciamiento y liderazgo en las comunidades educativas, lo que significa que estas mismas tienen que ser protagonistas de sus propios discursos y construir sus realidades, sin estar dependiendo de las acciones que vienen del exterior a su territorio. En esta perspectiva, el liderazgo integral educativo innovador tiene la capacidad de conjugar y articular las propias metodologías de la comunidad a partir de conocer profundamente la raíz de sus propios problemas.

El sujeto líder integral educativo innovador no enfatiza tanto en la tipología o estilos de liderazgo sino en aquellos aspectos que pueden viabilizar, de mejor forma, la estrategia asumida para el objetivo de la intervención en el territorio. Por ello, su mirada está puesta en las acciones que se le atribuyen al liderazgo y que, en nuestro caso, se definen por: el territorio, el PEI de las escuelas normales superiores, las competencias y el autoconocimiento que posee para hacer primar lo colectivo por encima de lo individual. Todos estos aspectos, asumidos desde los valores que serán diferentes dependiendo de la vida y los conflictos del contexto, lo que implica potenciar una epistemología que articule el conocimiento propio, con los demás y con el territorio.

Por último, no sobra señalar que los repertorios teóricos y prácticos ofrecidos para la sistematización de experiencias se traslucen en metodologías y diseños de propuestas que pueden ser valiosas para continuar reflexionando y revisando.

\section{Declaración de Material complementario}

Este artículo tiene disponible como material complementario:

- La versión preprint del artículo en https://doi.org/10.5281/zenodo.3351790

\section{Referencias}

Acosta Valdeleón, W. (2016). Innovación social educativa. Un camino a la transformación de las comunidades educativas. Editorial Redipe. 
Andere, E. (2017). Director de escuela en el siglo XXI. ¿Jardinero, pulpo o capitán? Siglo XXI.

Asensio Aguilera, J. M. (Enero-abril, 2002). Las actitudes en la reforma: Un aspecto de la educación emocional. Revista española de pedagogía, 60(221), 51-64. https://core.ac.uk/download/ pdf/41571643.pdf

Bárcena,F. y Mèlich, J.-C. (2000). La educación como acontecimiento ético. Natalidad, narración y hospitalidad. Paidós.

Bernal Martínez de Soria, A. e Ibarrola García, S. (2015). Liderazgo del profesor: Objetivo básico de la gestión educativa. Revista Iberoamericana de Educación, 67, 55-70. https://doi. org/10.35362/rie670205

Bolívar, A. (2010). El liderazgo educativo y su papel en la mejora: Una revisión actual de sus posibilidades y limitaciones. Psicoperspectivas. Individuo y Sociedad, 9(2), 9-33. https://doi. org/10.5027/psicoperspectivas-Vol9-Issue2-fulltext-112

Brito Lara, M., Chagoyán García, P. y Herrera Rendón, E. (2016). Liderazgo directivo o exitoso en la escuela secundaria. Proceso entre la formación profesional y la intuición. Boletín Redipe, 5(2), 55-61. https://dialnet.unirioja.es/servlet/articulo?codigo=6064477

Bustos Jiménez, A. (2006). Los grupos multigrado de educación primaria en Andalucía [Tesis doctoral]. Universidad de Granada, España. https://hera.ugr.es/tesisugr/16158933.pdf

Cifuentes Gil, R. M. (Ed.). (2017). Experiencias docentes en la universidad: Fundamentación y sistematización. Universidad de la Salle.

Congreso de la República de Colombia. (1994). Ley 115 de febrero 8 de 1994. Por la cual se expide la ley general de educación. https://www.mineducacion.gov.co/1621/articles-85906 archivo pdf.pdf

Cortina, A. (2009). Ciudadanos del mundo. Hacia una teoría de la ciudadanía (3. a ed.). Alianza.

Covey, S. R. (2013). El liderazgo centrado en principios. Paidós.

Gardner, H. (1993). Multiple intelligences. Basic Books.

Gardner, H. (1997). Les formes de l'intelligence. Odile Jacob.

Gómez Ortiz, R. A. (2006). El liderazgo empresarial para innovación tecnológica para las micro, pequeñas y medianas empresas. Universidad \& Empresa, 5(11), 62-91. http://www.redalyc. org/pdf/1872/187217476004.pdf

Larivée, S. (2010). Las inteligencias múltiples de Gardner. ¿Descubrimiento del siglo o simple rectitud política? Revista Mexicana de Investigación en Psicología, 2(2), 115-126. http:// www.medigraphic.com/pdfs/revmexinvpsi/mip-2010/mip102h.pdf

\begin{tabular}{l|l}
\hline 20 & Amparo Novoa-Palacios
\end{tabular}

Los artículos de la Revista Electrónica Educare del Centro de Investigación y Docencia en Educación de la Universidad Nacional, Costa Rica, se comparten bajo términos de la Licencia Creative Commons: Reconocimiento, No Comercial, Sin Obra Derivada 3.0 Costa Rica. Las autorizaciones adicionales a las aquí delimitadas se pueden obtener en el correo: educare@una.cr 
Lussier, R. N. y Achua C. (2002). Liderazgo. Teoría, aplicación y desarrollo de habilidades (2. ${ }^{\text {a ed.). }}$ Thomson Learning.

Lupano Perugini, M. L., y Castro Solano, A. (2006). Estudios sobre el liderazgo:Teorías y evaluación. Psicodebate.Psicología, Culturay Sociedad,6, 107-122.https://doi.org/10.18682/pd.v6i0.444

Meléndez de León, N. I. (1997). Liderazgo carismático. Cuaderno de investigación en la educación, 11, 26-35. https://revistas.upr.edu/index.php/educacion/article/view/16452/13996

Mèlich, J.-C. y Pelou, J. (Coords.). (2000). La veu de l'altre. Reflexions i experiènces per educar en valors ètics. Universitat Autónoma de Barcelona.

Mineducación (6 de abril de 2018). Las escuelas normales superiores y el Ministerio de Educación verifican las condiciones de calidad de los programas de formación complementaria. https:// www.mineducacion.gov.co/1759/w3-article-368491.html? noredirect=1

Ministerio de Educación Nacional. (2012). Manual para la formulación y ejecución de planes de educación rural. Calidad y equidad para la población de la zona rural. Autor. https://www. mineducacion.gov.co/1759/articles-329722 archivo pdf Manual.pdf

Novoa-Palacios, A. (2016). Innovación en la formación inicial de maestros en contextos rurales en Colombia: Normales superiores de Gigante, Huila y María Montessori en Bogotá (Proyecto de investigación CIV15101). Universidad de La Salle.

Portela Pruaño, A., Nieto Cano, J. M. y Toro Soriano, M. (2009). Historias de vida: Perspectiva y experiencia sobre exclusión e inclusión escolar. Profesorado. Revista de currículum y formación del profesorado, 13(9), 193-218. https://recyt.fecyt.es/index.php/profesorado/ article/view/42292/24235

Prats Gil, E. (2007). Retos y escenarios para la construcción de identidades múltiples: Una perspectiva pedagógica. En E. Prats Gil (Coord.), Multiculturalismo y educación para la equidad (pp. 121-152). Octaedro.

Real Academia Española. (2018). Diccionario de la lengua española. Autor. http://dle.rae. es/?id=0cWXkpX

Rodríguez Espinoza, S., Cárdenas Cofre, C. y Campos Vergara, F. (2012). El desarrollo del liderazgo educativo. Evidencias de un estudio de historias de vida. Revista lberoamericana sobre Calidad, Eficacia y Cambio en Educación, 10(1), 44-57. https://revistas.uam.es/index.php/ reice/article/view/3073/3285

Sánchez-Reyes, J. B. y Barraza-Barraza, L. (2015). Percepciones sobre liderazgo. Ra Ximhai, 11(4), 161-170. https://doi.org/10.35197/rx.11.01.e2.2015.10.js 
Savater, F. (1991). El valor de educar. Ariel.

Sierra Villamil, G. M. (2016). Liderazgo educativo en el siglo XXI, desde la perspectiva del emprendimiento sostenible. Revista Escuela de Administración de Negocios, 81, 111-128. https://doi.org/10.21158/01208160.n81.2016.1562

Vergara Alonso, M. H. (2012). Liderar en escuelas normales que aprenden. Responsabilidad y Calidad. Revista Iberoamericana para la Investigación y el Desarrollo Educativo, 3(5), 111 122. https://doi.org/10.23913/ride.v3i5.64

Weber, M. (2002) Economía y Sociedad. Esbozo de sociología comprensiva. Fondo de Cultura Económica. $\quad$ https://zoonpolitikonmx.files.wordpress.com/2014/08/max-webereconomia-y-sociedad.pdf 